\title{
Análise de acidentes fatais na mineração - o caso da mineração no Peru
}

\author{
(Analyzes of fatal accidents in the mining \\ industry - the case of Peruvian mining)
}

\section{Resumo}

A mineração é uma das atividades industriais que oferecem maior risco; embora nos últimos anos tenham-se percebido reduções na taxa de lesões e acidentes, o seu número e grau de severidade ainda são elevados. As causas fundamentais para as altas taxas de acidentes podem ser atribuídas às condições inseguras e os atos inseguros; nesse cenário, a identificação de problemas de segurança visando à proposta de soluções efetivas para gerenciar riscos faz-se necessária. Por outro lado, a dependência de países em desenvolvimento por indústrias primárias como a mineração é evidente. Na economia peruana, aproximadamente, $16 \%$ do PIB e mais de $50 \%$ das exportações referem-se a esta indústria, destacando sua posição competitiva na mineração mundial. Esse artigo analisa os acidentes fatais na mineração peruana, desde o ano 2000 até maio de 2008. A fonte de informação primária foi o registro de acidentes, disponibilizado pelo Ministério de Energia e Minas do Peru; identificando-se vários tipos de acidentes com destaque para aqueles provocados por queda de rochas em minas subterrâneas. A maioria das vitimas são trabalhadores de empresas terceirizadas prestadoras de serviços especializados. Os resultados mostram que a mineração subterrânea tem maiores riscos do que a de superfície.

Palavras-chave: Análise de acidentes, acidentes fatais, mineração peruana.

\section{Wildor Theodoro Hennies \\ Prof., Dr. Departamento de Engenharia de Minas e Petróleo da EPUSP E-mail:wildorth@usp.br}

\section{Wilson Iramina}

\section{Prof., Dr. Departamento de Engenharia de Minas e Petróleo da EPUSP \\ E-mail:wilsiram@usp.br}

\section{Juan Francisco Soto Elguera}

Prof., Departamento de Ingeniería de Minas de la Universidad Nacional de

San Antonio Abad del Cusco - Peru E-mail:fsoto@unsaac.edu.pe

\begin{abstract}
Mining is one of the industrial activities that offers greater risks; although in recent years, reduction in the rate of injuries and accidents has been observed, the numbers and degree of severity are high. The basic causes for the high rates can be attributed to unsafe conditions and unsafe acts; in this instance, the identification of security problems aiming to propose effective solutions becomes necessary for risk management. On the other hand, the dependence of developing countries on primary industries such as mining is evident. In the Peruvian economy, approximately $16 \%$ of the GIP and more than $50 \%$ of the exportations are contributed to this industry, showing its competitive position in worldwide mining. This article analyzes fatal accidents in Peru, from the year 2000 up to May of 2008. The primary information source was the fatal accident registry available at the Peruvian State Department of Energy and Mines. Various types of accidents were identified, with emphasis on those provoked by rock falls in
\end{abstract}


underground mines. The majority of the victims belong to contracted companies, rendering special services. The results show that underground mining has larger risks than surface mining.

Keywords: Analysis of accidents, fatal accidents, Peruvian mining.

\section{Introdução}

O setor mineiro é vital para muitos países e compreende o aproveitamento de minerais metálicos, não metálicos e combustíveis. O uso extensivo de matérias-primas minerais envolve sua aplicação na geração de energia elétrica, produção de cimento, aço, fertilizantes, materiais de construção, asfalto, produtos medicinais, entre outros. Historicamente, a mineração tem sido uma das atividades que apresentam maior risco no seu exercício.

A economia de países em desenvolvimento tem uma dependência significativa de atividades primárias como a indústria da mineração, pois uma parte significativa do PIB é contribuído por este setor; a mineração, também, é importante pelo potencial que tem para a geração de empregos, tanto diretos, como indiretos, em regiões que eram caracterizadas pela pobreza.

Por outra parte, os trabalhadores mineiros estão expostos a uma série de riscos, no local de trabalho, que podem estar associadas a acidentes provocados por choques elétricos, explosões, asfixia, queda de rochas, ruído, exposição ao pó, má iluminação, deficiência de ventilação entre outros; caracterizando a mineração como uma das atividades mais perigosas (Groves et al., 2007).

As causas fundamentais para a ocorrência de acidentes são as condições inseguras e os atos inseguros. As condições inseguras aparecem quando as considerações de projeto de mina são insuficientes, quando não são reconhecidas com antecedência as condições geológicas, quando existe deficiência na manutenção dos equipamentos, entre ou- tros. Atos inseguros aparecem, principalmente, em função de comportamento inadequado, alguns deles associados à falta de informação (Battacherjee, 1991).

Embora nos últimos anos tenhamse percebido reduções significativas na taxa de lesões e acidentes fatais na mineração, o número de acidentes e seu grau de severidade ainda são elevados, quando se compara a mineração com outras atividades industriais. Nesse cenário, a prevenção da saúde e a segurança dos trabalhadores, através da sua gestão adequada, são consideradas como ações estratégicas pela maioria dos governos.

No entanto, em países em vias de desenvolvimento, existe uma limitada informação sobre a ocorrência de acidentes, principalmente devido ao fato de que, na maioria das vezes, os acidentes sem resultados fatais não são declarados aos órgãos competentes, o que torna sua análise e compreensão mais difícil.

Tornar a atividade da mineração mais segura significa implementar um planejamento assim como implementar políticas de segurança, ações que deverão ser permanentemente monitoradas e controladas. As ações corporativas devem se basear em métodos e técnicas que controlem os riscos e, desse modo, possam reduzir os acidentes (Battacherjee, 1991).

Nesse cenário, a gestão de segurança ganhou importância e está sendo bastante reconhecida devido a gestão de segurança envolver vários aspectos, como o desenvolvimento de locais de trabalho mais seguros, melhor entendimento das condições do trabalho, aproveitamento das mudanças tecnológicas para se melhorem as condições de trabalho.

A análise de acidentes, através da sua classificação, tipificação, identificação de risco e seu grau de severidade se faz necessária, para que se criem medidas eficazes para o controle de acidentes (Hennies,1980). As decisões a serem tomadas, na gestão de segurança, devem centrar-se na escolha e na prioriza- ção de problemas ou nas áreas vulneráveis a acidentes. As decisões devem estar baseadas no reconhecimento de riscos encontrados em cada atividade que compreende o processo produtivo.

A gestão de segurança deverá ser praticada de maneira consistentes visando aos objetivos globais da organização. Para isso, deve procurar obter ótima alocação dos recursos e saber utilizá-los, obtendo as melhores soluções.

O presente artigo faz um estudo dos principais tipos de acidentes ocorridos na mineração peruana no período compreendido entre o ano 2000 e maio de 2008.

\section{A mineração peruana no cenário mundial}

A atividade da mineração é uma das mais importantes da economia peruana, representando, normalmente, mais de $50 \%$ das exportações, com cifras que superam os 15.000 milhões de dólares. Por sua própria natureza, a mineração de grande porte constitui o setor que gera maiores movimentos de capital. Atualmente a mineração contribui com, aproximadamente, $16 \%$ do PIB e constitui $62 \%$ das exportações (Mem, 2008), conforme mostrado na Tabela 1.

O Peru é um país de tradição mineira desde a época colonial com a explotação de minas como Potosí (hoje pertencente à Bolívia) e as minas de mercúrio de Santa Bárbara. Esse mercúrio, por sua vez, era utilizado para a amalgamação de minério de ouro, que era enviado para a Europa, particularmente para a Espanha. Entre os principais minerais que o Peru produz destacam-se: cobre, ouro, ferro, prata, zinco, chumbo, entre outros. Atualmente todos eles são bastante utilizados como insumos para processos industriais de alto nível tecnológico.

A atividade da mineração no Peru começou, industrialmente, com a explotação da mina de Cerro de Pasco, no departamento do mesmo nome, em 1905, e, em 1922, se inaugurou o complexo metalúrgico de La Oroya, no departamento 
Renan Collantes Candia et al.

de Junin. Na última década do século passado, foram outorgadas várias concessões mineiras como parte da política de abertura de mercados, política esta incentivada pelo governo de Alberto Fujimori. Entre as minas de maiores portes existentes no Peru destacam-se Antamina e Pierina (Ancash), Cuajone (Moquegua), Toquepala (Tacna), Cerro Verde (Arequipa), Tintaya (Cusco), San Rafael (Puno), Yanacocha e Sipan (Cajamarca), entre outras. Estima-se que o Peru tem $16 \%$ das reservas minerais do mundo. A maior parte dessas operações e projetos se encontra na faixa da cordilheira dos Andes (Figura 1).

As Tabelas 2 e 3 mostram as principais reservas estimadas dos principais minérios explotados e a posição na classificação mundial e na América Latina, respectivamente.

O Peru tem uma privilegiada posição competitiva na mineração mundial, mantendo uma liderança na América Latina e mundial. Em 2007, foi o maior produtor mundial de prata, zinco, estanho, bismuto, telúrio, chumbo, ouro e índio. Foi, também, o segundo maior produtor de cobre no mundo, perdendo apenas para o Chile, conforme mostrado na Tabela 3.

Os principais compradores de minerais do Peru são os Estados Unidos, a Suíça, o Reino Unido, o Japão, entre outros; assim desde 1990 a produção de minerais incrementou-se em aproximadamente $500 \%$. A privatização da mineração de grande porte desde 1991 atraiu mais de cem empresas estrangeirais, destacando-se as empresas canadenses, australianas, americanas, sul-africanas, mexicanas, suíças, inglesas, entre outras.

\section{Causas de acidentes na mineração}

A compreensão da gênese dos acidentes de trabalho é importante para o desenvolvimento das práticas de prevenção. Medidas como treinar, conscientizar, orientar, recomendar mais cuidado, advertir, usar equipamentos de proteção individual para se evitarem acidentes ficam carentes de sentido se não se iden-
Tabela 1 - Exportações peruanas FOB por grupo de produtos em 2007 (milhões de US\$) (MEM, 2008).

\begin{tabular}{l|c|c}
\hline 1. Produtos tradicionais & $\mathbf{2 1 , 4 9 3 . 0}$ & $\mathbf{( \% )}$ \\
\hline Pesqueiros & $1,456.0$ & $5 \%$ \\
\hline Agrícolas & 460.4 & $2 \%$ \\
\hline Minerais & $17,328.4$ & $62 \%$ \\
\hline Petróleo e derivados & $2,248.3$ & $\mathbf{8} \%$ \\
\hline 2. Produtos não tradicionais & $\mathbf{6 , 2 8 8 . 1}$ & $\mathbf{( \% )}$ \\
\hline Agropecuários & $1,503.3$ & $5 \%$ \\
\hline Pesqueiros & 497.6 & $\mathbf{2} \%$ \\
\hline Têxteis & $1,729.8$ & $6 \%$ \\
\hline Madeiras e papéis, e suas manufaturas & 360.4 & $1 \%$ \\
\hline Químicos & 803.3 & $3 \%$ \\
\hline Minerais não metálicos & 164.8 & $1 \%$ \\
\hline Sídero-metalúrgicos e joalharia & 906.6 & $3 \%$ \\
\hline Metal-mecânicos & $\mathbf{2 1 5 . 4}$ & $1 \%$ \\
\hline Outros 1* & 106.8 & $0 \%$ \\
\hline 3. Outros 2 ** & $\mathbf{1 7 4 . 5}$ & $1 \%$ \\
\hline 4. TOTAL EXPORTAÇÃo & $\mathbf{2 7 , 9 5 5 . 6}$ & $100 \%$ \\
\hline Inclui & &
\end{tabular}

* Inclui peles, coros, artesanato.

** Inclui venda de combustíveis e alimentos a naves estrangeiras, reparação de bens de capital.

Tabela 2 - Principais reservas provadas e prováveis de minérios (MEM, 2008).

\begin{tabular}{c|c|c}
\hline Minério & Unidade & Ano: 2006 \\
\hline Cobre & miles de TMF & 57,132 \\
\hline Ouro & miles de onzas finas & 72,823 \\
\hline Zinco & miles de TMF & 17,106 \\
\hline Prata & miles de onzas finas & $1,915,282$ \\
\hline Chumbo & miles de TMF & 6,295 \\
\hline Ferro & miles de TLF & $1,141,203$ \\
\hline Estanho & miles de TMF & 474 \\
\hline
\end{tabular}


tificam as causas dos acidentes dentro de uma organização.

As principais causas de acidentes de trabalho são as condições inseguras, os atos inseguros ou a combinação de ambos (Paul e Maiti, 2007). As condições inseguras aparecem através de projetos de mina inadequados, incertezas de caráter geológico-estruturais, condições inadequadas de manutenção de equipamentos, supervisão inadequada ou uma combinação desses fatores. Os atos inseguros se manifestam, principalmente, através de atitudes comportamentais consideradas como erradas e constituem direta ou indiretamente $90 \%$ dos acidentes de trabalho (Battacherjee, 1991).

Algumas das publicações mais recentes na investigação de acidentes de trabalho apontam para a crescente importância do papel dos fatores organizacionais como sendo determinantes para $o$ acontecimento de acidentes de trabalho. Tem-se investigado a importância dos fatores organizacionais na gênese da seqüência de situações de acidentes, identificando os processos perigosos, a pressão nas tarefas de produção e a falta de comunicação como sendo os maiores determinantes na ocorrência de acidentes (Wright, 1986).

A partir de estudos de situações de acidentes, no mar, para identificar os tipos de erros humanos, identificaram-se os fatores organizacionais tais como o processo de informação e a pressão social como sendo os fatores que influenciam o aspecto comportamental das pessoas (Wagenaar \& Groeneweg, 1987). Problemas relacionados à comunicação contribuem para o acontecimento de acidentes. Os eventos que causam um acidente são acumulativos pois foram ignorados ou interpretados erroneamente, sendo decorrentes de hábitos ou rotinas equivocados, de falsas expectativas, de comunicação deficiente, de procedimentos mal elaborados e de excesso de confiança. A gerência deve intervir nas boas práticas de segurança, através da criação de uma cultura positiva de segurança e de aprendizado. Em suas ações, a gerência deve criar espaço para que os erros e os acidentes sejam abertamente discutidos.

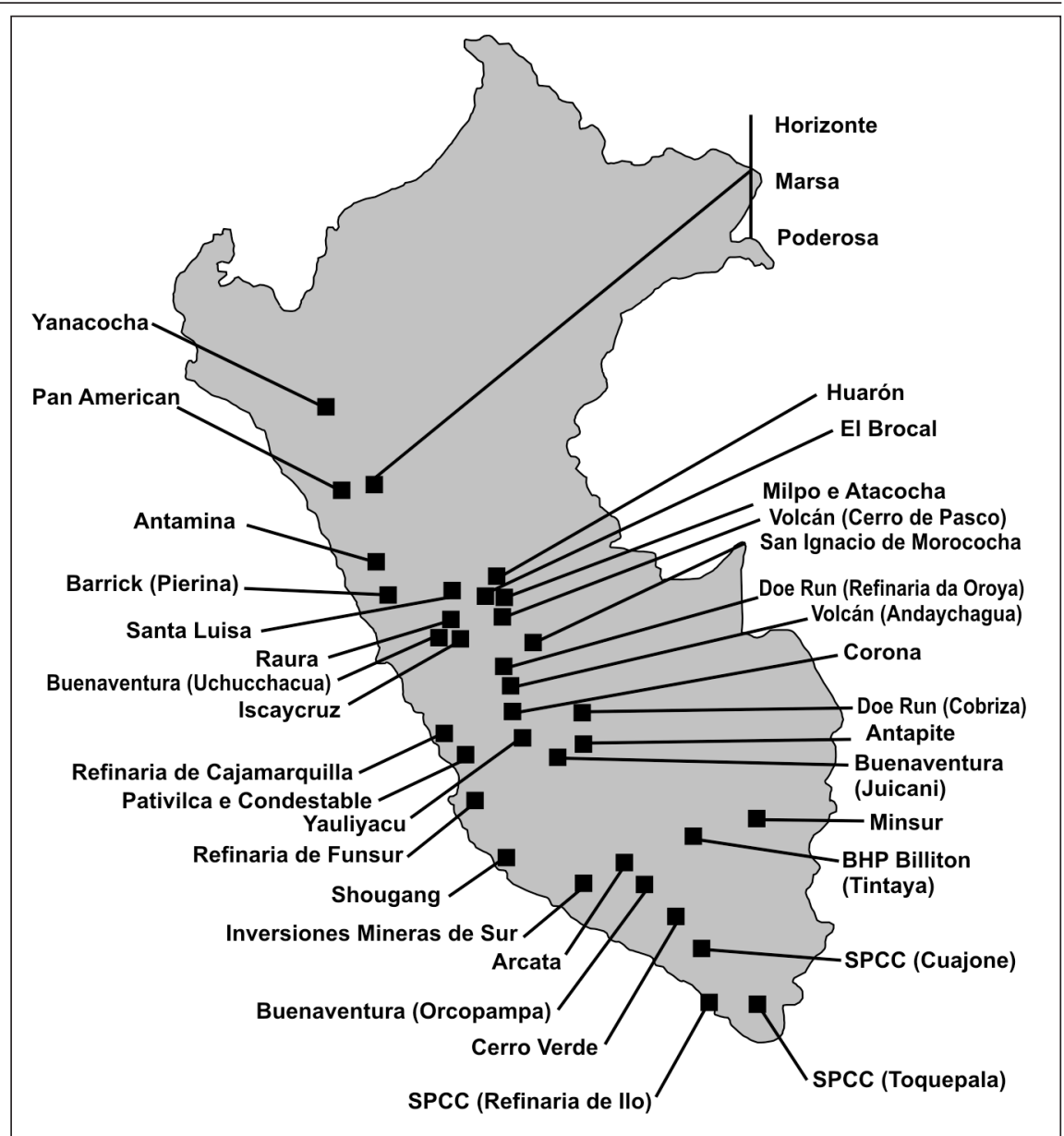

Figura 1 - Principais unidades mineiras do Peru.

Tabela 3 - Posição de produção mineral do Peru no ano 2007 (MEM, 2008).

\begin{tabular}{c|c|c}
\hline Mineral & Posição mundial & Posição na América Latina \\
\hline Prata & 1 & 1 \\
\hline Zinco & 2 & 1 \\
\hline Estanho & 3 & 1 \\
\hline Bismuto & 3 & 1 \\
\hline Telúrio & 3 & 1 \\
\hline Chumbo & 4 & 1 \\
\hline Ouro & 5 & 1 \\
\hline Índio & 8 & 1 \\
\hline Cobre & 2 & 2 \\
\hline Molibdênio & 4 & 2 \\
\hline Selênio & 7 & 2 \\
\hline Ferro & 17 & 5 \\
\hline
\end{tabular}


Alguns autores estabeleceram que os acidentes são conseqüência de três fatores: humanos, organizacionais e tecnológicos (Paul \& Maiti, 2007). Foram, ainda, identificados fatores-chave para um bom desenvolvimento da segurança na organização: balanço entre a segurança e a produção, pressão do tempo, sistemas de coordenação e comunicação e cultura de segurança (Emprey, 1992).

As medidas de segurança têm de levar em consideração inúmeros fatores, destacando-se a idéia de que determinados aspectos organizacionais influenciam diretamente o comportamento dos trabalhadores. Assim a gerência de segurança deve estar atenta para a relação entre os processos de trabalho e seus riscos, para a necessidade de incrementação da cooperação dos trabalhadores entre si e à empresa, e para a necessidade de se levar em consideração a experiência dos trabalhadores. Essa gama de observações levará a gerência de segurança a implementar uma eficaz política na referida área (Simard \& Marchand, 1995).

As teorias de causalidade de acidentes podem ser agrupadas em seis modelos conceituais (Almeida, 1995):

- Cadeia de múltiplos eventos que descreve uma sequência temporal de eventos que conduzem ao acidente, entendendo-se que sua origem é multicausal.

- Modelo epidemiológico; apresenta o acidente como sendo o resultado da interação entre as variáveis da pessoa, do agente (equipamentos, ferramentas, máquinas) e do ambiente de trabalho (físico e social).

- Modelo de troca de energia; indica que os acidentes são causados por alguma forma de energia, que é o "agente".

- Modelo comportamental; compreende duas categorias. A primeira valoriza a existência de situações nas quais deve de ser tomada uma decisão para a ação na presença de um risco e a segunda defende a existência de modelo comportamental de propensão ao acidente, segundo essa ideía, algumas pessoas teriam predisposição inata a maior acidentalidade.
- Modelo sistêmico; aborda o acidente como o resultado extremo no sistema homem-máquina, salientando a interação entre seus componentes. Situações de sobrecarga e de erros no sistema podem conduzir à quebra de seu equilíbrio, conseqüentemente, à ocorrência de acidente.

Contudo é evidente que, na ocorrência de acidentes, concorrem na maioria das vezes, vários fatores. para se fazer uma análise sobre a ocorrência de acidente de trabalho, muitos faotres têm de ser levados em consideração. Há um sinergismo de causas e todas devem ser analisadas de forma criteriosa. A análise deve, portanto, identificar os fatores presentes na origem do perigo, bem como os fatores que desencadeiam ou liberam aquele perigo em potencial, assim como as condições do sistema envolvido.

Por outro lado as principais causas dos acidentes na mineração são (MSHA, 1997):

- Eletricidade.

- Enclausuramento em espaços confinados.

- Explosão de explosivos manufaturados.

- Explosões de dutos sob pressão.

- Queda e deslizamento de materiais.

- Queda da face, suporte ou pilar da escavação.

- Queda de rochas do teto da escavação.

- Incêndios.

- Manuseio de materiais.

- Ferramentas manuais.

- Carregamento de minério.

- Transporte horizontal e vertical.

- Ignição ou explosão de gases ou poeira.

- Inundação.

- Equipamentos e máquinas.

\section{Metodologia}

As informações sobre acidentes fatais foram obtidas de fontes oficias do Ministério de Energia e Minas do Peru
(MEM, 2008), sendo que tais informações foram utilizadas como fontes primárias. Essas informações encontram-se em formato pdf e Excel e, para seu tratamento, foram adequadamente organizadas e armazenadas em formato padrão Excel. Foram analisados os acidentes fatais envolvendo trabalhadores do setor da mineração peruana acontecidos no período compreendido entre os anos 2000 e maio de 2008.

Os acidentes fatais são definidos como aqueles que provocam a morte. Tal acidente ocorre com qualquer indivíduo que, no momento do acidente, estava desenvolvendo trabalhos no setor da mineração, o que quer dizer que, na população em estudo, estão compreendidos, tanto funcionários da empresa de mineração, como funcionários de empresas prestadoras de serviços especializados.

Essa definição envolve os trabalhadores envolvidos na extração de minerais em operações, tanto a céu aberto, como subterrâneas, através das diversas operações unitárias de lavra, assim como nas operações ou serviços auxiliares. Ainda foram incluídos, nessa categoria, os trabalhadores envolvidos no beneficiamento de minérios, assim como atividades de embarque no porto.

Os fatores causais para o acontecimento de acidentes foram definidos como aqueles que contribuíram diretamente para o acontecimento do acidente fatal. Foram vários os fatores identificados como sendo os contribuintes diretos. A classificação dos fatores envolvidos na ocorrência de acidentes foi feita segundo critérios da MSHA; no entanto, é importante ressaltar que, na maioria das vezes, são vários os fatores envolvidos na ocorrência de acidentes.

Para o tratamento estatístico descritivo da informação, foi utilizado o pacote Excel da Microsoft ${ }^{\circledR}$. Ainda a análise dos principais tipos de acidentes fatais, seu comportamento no tempo, os tipos de acidente mais representativos, como sua incidência, são apresentados em forma de tabelas e gráficos. 
Análise de acidentes fatais na mineração - o caso da mineração no Peru

\section{Resultados}

Durante o período de análise, isto é, desde o ano 2000 até maio de 2008, aconteceram 471 acidentes fatais com 531 vítimas. $O$ tipo de acidente mais representativo foi a queda de rochas em escavações subterrâneas $(36,53 \%)$, seguido pelo trânsito de pessoas $(9,23 \%)$, pela queda de pessoas $(9,04 \%)$, pelo escorregamento de terreno $(7,91 \%)$, entre outros, conforme mostrado na Tabela 4.

A média de acidentes fatais, por ano, foi de 52, com uma média de 59 vítimas, sendo que 175 vítimas foram trabalhadores da empresa mineira e os restantes 356 pertenciam a empresas prestadoras de serviços especializados (empreiteiras), o que corresponde a $33 \%$ e $67 \%$ das vítimas fatais, respectivamente, conforme mostrado na Figura 2.

A análise dos resultados mostra, também, que, no período, o número de acidentes fatais, na mineração subterrânea, de superfície, no beneficiamento e embarque de minerais, foi de 426, 38 e 7; gerando 482, 42 e 7 vítimas, respectivamente. As Figuras 3 e 4 mostram as participações percentuais de acidentes e vítimas fatais segundo o tipo de atividade.
Tabela 4 - Número de vítimas em acidentes fatais entre o ano 2000 e maio de 2008.

\begin{tabular}{c|c|c}
\hline Tipo de acidente & Quantidade & $\%$ \\
\hline Queda de rochas & 194 & 36,53 \\
\hline Escorregamento de terreno & 42 & 7,91 \\
\hline Afogamento ou inundação & 6 & 1,13 \\
\hline Intoxicação, asfixia, absorção e radiações & 38 & 7,16 \\
\hline Trânsito & 49 & 9,23 \\
\hline Carregamento e transporte & 30 & 5,65 \\
\hline Manobra de equipamentos & 24 & 4,52 \\
\hline Enterramento por afundamento de terreno & 6 & 1,13 \\
\hline Manipulação de materiais & 9 & 1,69 \\
\hline Queda de pessoas & 48 & 9,04 \\
\hline Explosivos & 23 & 4,33 \\
\hline Ferramentas & 2 & 0,38 \\
\hline Energia elétrica & 19 & 3,58 \\
\hline Outros & 41 & 7,72 \\
\hline ToTAL & 531 & 100 \\
\hline
\end{tabular}

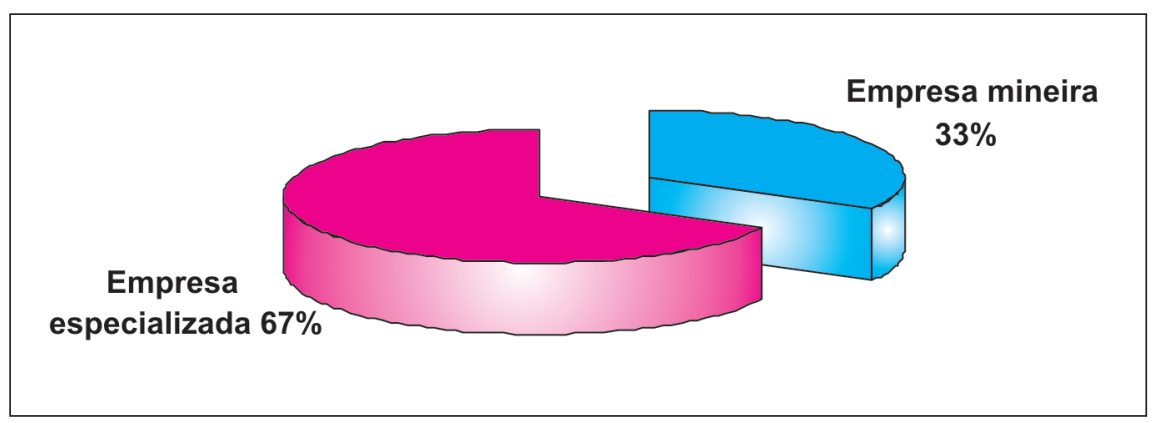

Figura 2 - Participação percentual de vitimas fatais segundo vinculo empregatício.

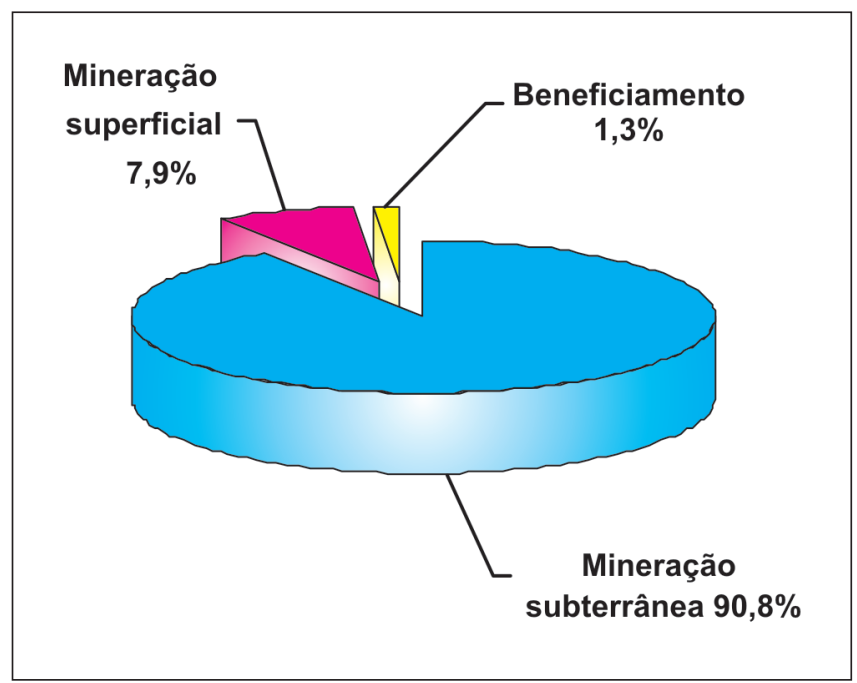

Figura 4 - Participação percentual de vítimas fatais segundo o tipo de atividade.
Figura 3 - Participação percentual de acidentes fatais segundo o tipo de atividade.

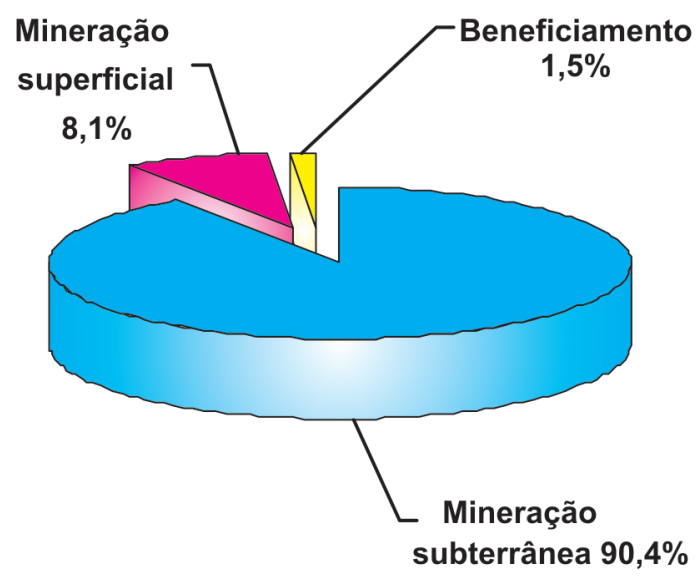




\section{Conclusões}

A atividade da mineração é uma das mais importantes na economia peruana, tendo uma posição privilegiada dentro do contexto mundial; no entanto, embora nos últimos anos tenham-se percebido melhorias significativas no controle de condições e atos inseguros, as incidências de acidentes fatais ainda são elevadas. A análise de acidentes fatais ocorridos entre o ano 2000 e maio de 2008 mostra que os tipos de acidentes mais representativos são aqueles causados por queda de rochas em escavações subterrâneas, seguido pelo trânsito de pessoas, pela queda de pessoas, pelo escorregamento de terreno e por intoxicações. $\mathrm{O}$ estudo mostra, ainda, que a maior parcela de acidentes acontece na mineração subterrânea, reforçando a tese de que esta é mais perigosa que a de superfície. A maioria das vítimas fatais pertenciam a empresas especializadas, que prestam serviço para a empresa mineira, mostrando que boa parte das operações de lavra assim como as operações auxiliares na indústria da mineração do Peru estão terceirizadas. Nesse cenário, faz-se necessária uma proposta de soluções efetivas para gerenciar riscos, visando a reduzir a taxa de acidentes fatais.

\section{Referências bibliográficas}

ALMEIDA, M.I. Desvendando a zona de sombras dos acidentes de trabalho. São Paulo: Departamento de Saúde Ambiental da Faculdade de Saúde Pública da Universidade de São Paulo - USP, 1995. 137 p. (Dissertação de Mestrado).

BATTACHERJEE, A. Mine safety managemnet: An aplication of risk analyses, forecasting techniques, and Markov process to injury experience data. The Pennsilvania State University, 1991. 301 p. (Doctoral Thesis).

EMPREY, D.E. Incoporating management and organizational factors into probabilistic safety assesment. Reliability Engineering and System Safety, n. 38, p. 199-208, 1992.

GROVES et al. Analyses of fatalities and injuries involving mining equipment. Journal of Safety Research., n. 38, p. 461-470, 2007.

HENNIES, W. T. Segurança na mineração e no uso de explosivos. São Paulo: Fundacentro, 1980. $45 \mathrm{p}$

MEM - MINISTERIO DE ENERGIA Y MINAS DEL PERU. Anexo estadistico del Peru. Disponível em:http://www.minem.gob.pe/archivos/dgm/estadisticas. Acesso em junho de 2008.

MINE SAFETY AND HEALTH ADMINISTRATION (MSHA). Injury experience in coal mining, 1996. Department of labor, Mine Safety and Health Administration, office of Injury and employment information, IR 1253. p5-7. Denver, CO. 1997.

PAUL, P. S., MAITI, J. The role of behavioral factors on safety management in underground mines. Safety Science, n. 45, p. 449-471, 2007.

SIMARD, M., MARCHAND, A. A multilevel analysis of organizational factors related to the taking of safety initiatives by work groups. Safety Science, n. 21, p. 113-129, 1995.

WAGENAAR, W.A., GROENEWEG, J. Accidents at sea: multiple causes and imposible consequances. International Journal of Man - Machine Studies, n. 27, p. 587-598, 1987.

WRIGHT, C. Routine deaths: fatal accidents in the oil industry. Sociological Review, n. 4, p. 265-289, 1986.

Artigo recebido em 31/10/2008 e aprovado em 22/05/2009.

\section{A REM tem novo endereço: FUNDAÇÃO GORCEIX - REM Rua Carlos Walter Marinho Campos, 57 Bairro: Vila Itacolomy 35400-000 - Ouro Preto - MG (31) $3551-4730 \quad$ (31) $3559-7408$ www.rem.com.br}

